\title{
Diabetic Patients' Quality of Life and Their Relationship in Compliance with Antidiabetic Treatment. Case Study of Patients in a Public Hospital in Greece
}

\author{
CHARALAMPOS PLATIS ${ }^{1 \mathrm{a}}$, ADAMANTIA SPANOU $^{1 \mathrm{~b}}$, PANTELIS MESSAROPOULOS $^{2 \mathrm{c}}$, \\ CATHERINE KASTANIOTI ${ }^{3 \mathrm{~d}}$, EMMANOUIL A. ZOULIAS $^{4 \mathrm{e}}$ \\ ${ }^{1}$ School of Social Sciences, Postgraduate Program in Health Care Management, \\ Hellenic Open University, Patra, GREECE \\ ${ }^{2} 3$ rd Department of Obsterrics and Gynaecology, National and Kapodistrian University of Athens, \\ Athens, GREECE \\ ${ }^{3}$ Department of Business Administration, University of Peloponnese, \\ Kalamata, GREECE \\ ${ }^{4}$ Faculty of Nursing, School of Health Sciences, National and Kapodistrian University of Athens, \\ Athens, GREECE \\ a)charisplatis@gmail.com, b) std106228@ac.eap.gr, ${ }^{c}$ pantelismess@gmail.com, \\ d)catherinekastanioti@yahoo.gr, ezoulias@nurs.uoa.gr
}

\begin{abstract}
Background \& Aim: Diabetes is a chronic disease that affects nearly half a million people worldwide. Although advances in technology and medical science have made diabetes management easier today, self-care and adherence to treatment (medication, diet, exercise, etc.) remain a major challenge for diabetic patients. The purpose of this study is to study the quality of life of patients with type 2 diabetes, their levels of compliance with antidiabetic treatment, and the relationship between the two.

Methods \& Materials: The present work followed the quantitative approach. A well-structured and reliable questionnaire used in a previous study was distributed to patients with type 2 diabetes mellitus. The results were analysed in SPSS statistical program and the correlation between antidiabetic treatment and quality of life was examined using Pearson coefficient.

Results: The study involved 165 patients with type 2 diabetes who were hospitalized at the General Hospital of Messinia. Among the dimensions of adherence to treatment, only personal care behaviour $(p<0.05)$ and adherence to care $(\mathrm{p}<0.05)$ were significantly and positively correlated with the physical quality of life health.

Conclusion: Patients with type 2 diabetes have moderate the low quality of life, in line with previous research findings. Lower extremity care compliance was associated with the quality of life of diabetic patients with physical health, although previous studies have generally found that compliance with a diet regimen as well as adherence to medication is associated with the quality of life of diabetic patients. Future research needs to address the same issue by taking larger and more representative samples.
\end{abstract}

Key-Words: - Diabetes mellitus, quality of life, antidiabetic treatment, treatment compliance

Received: October 3, 2019. Revised: February 2, 2020. Accepted: February 20, 2020. Published: March 18, 2020.

\section{Introduction}

\subsection{Background/Rationale}

Diabetes mellitus is a chronic disease due to insufficient insulin production by the pancreas or by the ineffectiveness of the insulin produced [1]. Type II diabetes mellitus is the most common type of diabetes today and characterized by a ser ies of malfunctions such as insulin resistance, the inadequate secretion of insulin and the excessive or inappropriate glucagon secretion. This type of diabetes affects mainly adults. The second most common type of diabetes is the type I diabetes mellitus, which mainly affects children and teenagers [2]. Diabetes mellitus affects today $8.5 \%$ of the world population and is the 8 th main death reason worldwide [1].

The medical management of diabetes mellitus type II is lifelong. The treatment requires medication and insulin, depending on the case, for the prevention or the delay of appearance or the effective management of life-threatening complications [3]. Important aspects of the medical treatment and management of diabetes mellitus are the daily monitoring of blood glucose, the healthy diet and the participation in physical activity and exercise. In addition to that regular monitoring, foot care, smoking cessation and regular medical visits 
are also important components of diabetes management [4].

In chronic diseases, such as d iabetes mellitus, compliance to treatment is critical for disease control, as non-compliance will often lead to inadequate management, deterioration of health, and uncertain expectations about treatment [5]. The reported incidence of inadequate compliance to medication in patients with type II diabetes mellitus ranges from $38 \%$ to $93 \%$ [6].

Non-compliance to antidiabetic treatment affects the health level of diabetic patients. According to Cheung et al. [7] estimated that less than 1 in 2 diabetic patients will achieve glycaemic goals and 2 in 3 will die prematurely due to the development of complications [7]. Patients who are less adherent to treatment are more likely to develop complications due to poor glycaemic control [8], [9] as well as are more likely to develop mobility problems and difficulties in self-care, daily activities, experience increased levels of pain and anxiety [10] and experience various mental health problems [11]. All the above mentioned contributing to lowering the quality of life associated with both physical and mental health.

The prevalence of diabetes estimated at $4.11 \%$ for people aged 20-70 in Greece. Studies in Greece have assessed the quality of diabetic patient's life and compliance with antidiabetic treatment, concluding in conflicting results. In a study by Avramopoulos et. al. [12], in various hospitals in the country, evaluated the glycaemic control, quality of life, and satisfaction with the treatment of patients with diabetes. A significant proportion of patients did not achieve glycaemic target levels and reported high levels of satisfaction with treatment. On the other hand [13] found that patients with type II diabetes in a public hospital showed good levels of compliance with treatment, but nevertheless reported high quality of life. Other international researches revealed the opposite results. It should be noted, however, that there have been studies that have shown opposite results. In the study of Martínez et al. [14], who evaluated the relationship between quality of life and medication adherence in a sample of 238 patients with type 2 diabetes, could not find any statistically significant correlation between the two parameters. Other demographic and psychosocial factors were associated with quality of life, such as patients' personal behaviour and good knowledge of diabetes mellitus. Furthermore it is quite interesting that a lot of technics based on mathematical models have been proposed in order to evaluate treatment effectiveness for diabetic patients[15], [16].

\subsection{Objectives}

Although international research indicates a correlation between the quality of life and compliance to antidiabetic treatment in patients with diabetes mellitus, this issue has not been sufficiently investigated up today among diabetic patients in Greece. The main objective of this work is to investigate if the findings of international research can extend to the Greek therapeutic reality.

The specific purpose of this work is to study the existence of any possible correlation between compliance to antidiabetic treatment and quality of life for diabetic patients in Greece. Based on the above-mentioned aim, the research questions under investigation are: 1) the identification and mapping of compliance levels with the anti-diabetic treatment for patients with type II diabetes, 2) the study of life quality related to patients physical and mental health with type II diabetes and 3) the study of whether and to what extent the patients quality of life with type II diabetes, is related to their degree of compliance to their antidiabetic treatment.

\section{Problem Formulation}

\subsection{Study Design}

In this work used a quantitative approach for the analysis. The questionnaire used in this work for the evaluation of Mellitus treatment was designed by [4]. This questionnaire has a high level of reliability, high level of structural integrity, adequate specificity and sensitivity to change. The SF-12 scale used for the evaluation of the patient's quality of life. The data collected and answers analysed using the SPSS statistical software. A descriptive statistical analysis performed as an initial approach. Analysis of possible correlations between quality of life and compliance with antidiabetic treatment. The correlation factor of Pearson used as a statistical measure of possible correlations.

\subsection{Setting}

The research performed on $165 \mathrm{pa}$ tients of General Hospital of Messinia during June 2019. The patients approached by the researcher, who explained the aim of the research. Patients asked for their consent in order to participate in the research. The research protocol, the questionnaire submitted for approval to the scientific council of the hospital, as the law obliges. The council replied positively with the decision number 8/31-5-2019. 


\subsection{Participants - Study Size}

As we mentioned above the research involved a total of 165 patients who were found at the Hospital of Kalamata. Furthermore, this sample considered as representative regarding the population of diabetic patients in this specific area of South Greece. Based on demographics, the majority of patients in this research were men $(57.6 \%)$, Greek origin $(97.6 \%)$, living in a city $(65.9 \%)$, primary school graduates $(32.3 \%)$ and married $(76.8 \%)$, had a monthly income of 601 to 100 euros $(64.6 \%)$ and were retired $(46.1 \%)$. The mean age of the sample was 64.82 years (standard deviation 10.49 and range 34 91 years), with most patients being 60 to 80 years old.

\subsection{Statistical Methods}

This investigation is concerned with both consistent and rational treatment of medication and compliance to the recommendations of a correlation between patient's quality of life with diabetes and compliance to antidiabetic treatment in the dimensions of medication administration and compliance to these recommendations; care (exercise, nutrition, blood sugar control and medication).

This work followed a quantitative approach using a well-structured questionnaire. The questionnaire used in the survey to assess compliance to antidiabetic treatment was the one developed by Intas [4], which has demonstrated good levels of reliability, structural validity, discernment and good sensitivity to change. The SF-12 scale used to assess diabetic patients' quality of life. The results of the questionnaires were collected and analysed using SPSS statistical software and presented using frequencies and descriptive statistics. The correlation between the quality of life and compliance to antidiabetic treatment was tested by the Pearson correlation coefficient.

\section{Problem Solution}

Patients' behaviours regarding antidiabetic treatment were initially evaluated to diet, exercise, lower limb care, blood sugar control, smoking cutting and general self-care actions of diabetes. Patients reported that they often followed a healthy diet during last week (mean $=4.24)$ and that they rarely consumed high-fat foods (mean $=1.87)$. Also, sometimes during the last week, they spent 30 consecutive minutes of physical activity (mean $=$ 3.29 ), but very rarely did intense exercise (mean $=$ 0.91 ). In addition to that during the last week quite often, they checked their blood sugar as directed by their doctor (mean $=4.14$ ) and very often, they checked their lower extremities (mean $=4.91$ ). Regarding smoking habits, 25.9\% were smokers. Overall, it was found that patients adopt moderately to some extent appropriate behaviour in their meals $($ mean $=3.62)$ and control of sugar $($ mean $=3.96)$, while to a large extent adopt an appropriate control plan of lower limbs care $($ mean $=4.87)$. On the contrary, to a small extent, they adopt proper behaviour in their exercise $($ mean $=2.10)$.

The table followed summarises the findings regarding the body characteristics of the sample. The analysis reveals that the average weight of the patients was $86.35 \mathrm{~K}$ gs (SD $=17.12)$, the mean height of 1.66 metres $(\mathrm{SD}=$ $0.09)$ and the mean BMI $31.29(\mathrm{SD}=5.45)$.

Table 1. Sample's Body Characteristics

\begin{tabular}{lcccc}
\hline \hline & Mean & SD & Min & Max \\
& & & & \\
\hline Weight & 86.35 & 17.12 & 45.00 & 148.00 \\
Height & 1.66 & 0.09 & 1.44 & 1.91 \\
BMI & 31.29 & 5.45 & 19.74 & 49.95 \\
\hline \hline
\end{tabular}

The median time since diagnosis of diabetes was 10.13 years (range 0-47 years), while the most reported comorbidities were hyperlipidaemia $(58.2 \%)$ and hypertension $(50.9 \%)$. This is illustrated also in the bar diagram below.

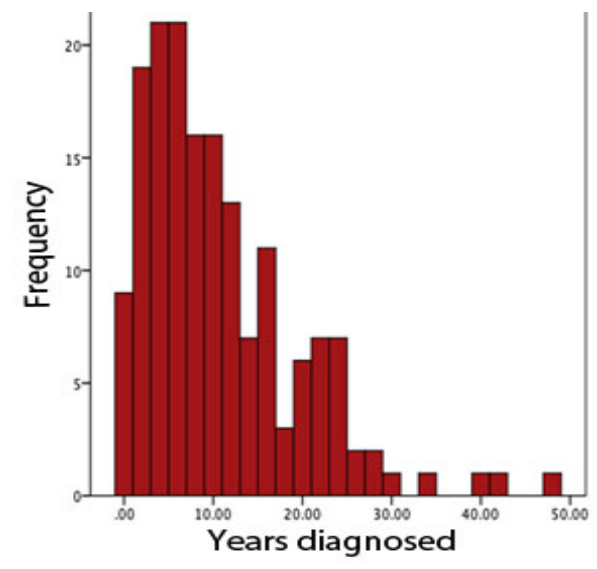

Fig. 1 Median time since diagnosis of diabetes

Self-care recommendations were also evaluated. Some patients, up to the percentage of $37.3 \%$, had received recommendations for a low-fat diet. The $29.8 \%$ had adopted a specific exercise plan (type, duration, intensity), $96.3 \%$ of patients had been given glucose control using a machine and 59.3\% 
had received recommendations for anti-diabetic pills and tablets.

Overall for patients' compliance with antidiabetic treatment, it was shown that patients adhere very little to a diet, but on the contrary, adhere to a highlevel medication (mean $=4.77$ ) and lower extremities care $($ mean $=4.94)$.

The quality of life of the sample patients was then evaluated. $34.5 \%$ of patients said their general health was good. However, $32.7 \%$ of patients said that their current state of health restricts them to some activities, while $80 \%$ of patients felt that their health condition forced them to do less than they would have liked. $67.9 \%$ thought that because of some emotional problem, they did less than they would like. $34.5 \%$ also found that pain significantly or significantly affected their usual work (work, home). Regarding mental health, $35.8 \%$ of patients said they felt very little or no peace in the last 4 weeks and $34.5 \%$ felt hopelessness and melancholy constantly or very often in the last 4 weeks. Also, $44.3 \%$ of patients felt little or no activity in the last 4 weeks and $29.1 \%$ said they were constantly or very often affected by their social activities due to their physical or mental health. Overall the results of the quality of life assessment showed that patients with type 2 diabetes who participated in the study had moderate to low levels of quality of life-related to physical and mental health.

The Pearson correlation coefficient used to investigate the possible association of quality of life with adherence to antidiabetic treatment. The results showed that personal attitudes towards lower extremity care were positively related to patients' physical health $(\mathrm{p}<0.05)$ and similarly, lower extremity care compliance was positively related to the quality of life-related to the physical health of patients $(p<0.05)$. However, no $c$ orrelations were found between other dimensions of compliance with antidiabetic treatment and other dimensions of quality of life related to physical and mental health.

Table 2. Statistical Analysis

\begin{tabular}{lcc}
\hline \hline & $\begin{array}{c}\text { Body Health } \\
\text { Pearson r / p }\end{array}$ & $\begin{array}{c}\text { Mental Health } \\
\text { Pearson r / p }\end{array}$ \\
\hline \multicolumn{3}{c}{ Personal Behaviour } \\
\hline Diet & $-.018 / .820$ & $-.059 / .463$ \\
\hline Exercise & $-.008 / .915$ & $-.002 / .975$ \\
\hline $\begin{array}{l}\text { Blood } \\
\begin{array}{l}\text { Glucose } \\
\text { check }\end{array}\end{array}$ & $-.113 / .155$ & $-.077 / .329$ \\
\hline $\begin{array}{l}\text { Lower } \\
\text { extremities }\end{array}$ & $.220 / .005$ & $-.025 / .755$ \\
\hline
\end{tabular}

\begin{tabular}{lll}
\hline \multicolumn{3}{c}{ Adherence } \\
\hline Diet & $.033 / .681$ & $-.049 / .540$ \\
\hline $\begin{array}{l}\text { Pharmaceu } \\
\text { tical } \\
\text { Treatment }\end{array}$ & $.124 / .119$ & $-.018 / .822$ \\
\hline $\begin{array}{l}\text { Lower } \\
\text { extremities }\end{array}$ & $.211 / .007$ & $.004 / .956$ \\
\hline Diet & $.030 / .701$ & $.049 / .536$ \\
\hline \multicolumn{3}{c}{ Recommendations } \\
\hline Exercise & $.177 / .025$ & $.113 / .155$ \\
\hline $\begin{array}{l}\text { Blood } \\
\text { Glucose } \\
\text { check }\end{array}$ & $.104 / .189$ & $.099 / .210$ \\
\hline $\begin{array}{l}\text { Pharmaceu } \\
\text { tical }\end{array}$ & $-.045 / .573$ & $.047 / .551$ \\
Treatment & & \\
\hline
\end{tabular}

Overall the results of the study showed that patients with type 2 diabetes exhibited low levels of diet compliance and high levels of lower extremity care and medication adherence. This result does not coincide with the findings of previous research in Greece, which showed higher rates of diet compliance [13].

The quality of life of diabetic patients in the study also appears to be affected, as overall moderate to low levels of physical and mental health-related quality of life were recorded. Similarly, several previous studies have found the low quality of life in patients with type 2 diabetes [17].

In the present study, only positive associations were observed between personal attitudes towards lower extremity care and compliance with lower extremity care with the quality of life associated with physical health. Although this result is consistent with the findings of Zioga et al. [13], this study also found other correlations between all dimensions of diabetes compliance (diet, exercise, blood sugar measurement, self-care recommendations) and all dimensions of quality of life, as assessed by the SF-36 scale, which were not identified in the present study due to possible differences in the samples between the two studies, but also possibly due to differences in the data collection tool for adherence to the antidiabetic treatment. Possible, some other factors may contribute to the quality of life of sample diabetes patients, such as complications of diabetes, type of medication, or duration of diabetes, but these associations were not examined in the present study. 


\section{Conclusion}

The present empirical study examined adherence to antidiabetic treatment and its association with quality of life in patients with type 2 diabetes, taking a sample of patients from a public hospital. Most patients reported regular visits to their physician, as about 1 in 3 had visited him 4 times in the last year, while 1 in 4 had visited him more than 4 times or up to 3 times in the last year.

The analysis of the results on patient behaviour reveals the adoption of a diet plan and the avoidance of high-fat foods, blood sugar control and lower extremity care. On the contrary, patients were less likely to follow an exercise program and rarely did vigorous exercise, although they sometimes reported that they spent 30 consecutive minutes of physical activity. Regarding the adoption of a healthy lifestyle in terms of smoking, about 1 in 2 patients reported that they were smoking.

Regarding self-care recommendations, it was found that less than 1 in 2 had received low-fat diet recommendations, while less than 1 in 3 had received recommendations for reducing their daily calories for weight loss and less than 1 in 5 had received recommendations for consumption of fruits and vegetables. Regarding exercise recommendations, less than 1 in 2 had received recommendations for gentle daily exercise, less than 1 in 3 had received recommendations for a specific type, duration, and degree of exercise, and about 1 in 4 had received recommendations for some daily exercise. Most patients had received recommendations for blood sugar control. Regarding medication, more than 1 in 2 ha $d$ received recommendations for anti-diabetic pills and tablets, while others had received recommendations for other medication (usually for insulin use). Regarding smoking recommendations, it appears that less than 1 in 4 were asked by their doctor if they smoke and similarly received smoking cessation recommendations.

Regarding the patients' quality of life, only 1 in 5 said it was very good or excellent, while the majority reported moderate to good quality of life. In the dimension of physical functioning 1 in 3 reported that their current state of health restricts the exercise of moderate-intensity activities to a significant extent and a greater proportion appears to be restricted when climbing stairs. The physical role of diabetic patients seems to be significantly affected by the illness, as a large proportion of patients report that their health status has forced them to do less than they would like and restricts them to work or types of other activities they would like to do. The emotional role of diabetes mellitus patients also seems to be significantly affected, as more than 2 out of 3 stated that because of an emotional problem they did less than they would like and were forced to do their job or activities less than they thought they would. Low or high quality of life seems to be also recorded in the dimension of physical pain, as over 2 in 3 reported that pain had moderately, to a great extent or very much affected their usual work. In the mental health dimension, more than 1 in 3 reported that they had rarely or never experienced serenity and serenity in the last 4 weeks and that they had been feeling hopelessly or very often in the last 4 weeks. In the area of vitality, almost $1 \mathrm{i} \mathrm{n} 2 \mathrm{f}$ elt short-lived or sometimes energetic. Finally, in the area of social functionality, almost 1 in 2 reported that the disease affected their social activities.

An overall assessment of the quality of life of diabetic patients indicates that they have moderate to low levels of quality of life-related to physical and mental health. These results appear to coincide with the findings of previous studies in diabetic patients in Greece. For example, Papadopoulos et al. [17] found low levels of quality of life in diabetic patients, especially in physical function, which were related, to factors such as duration of diabetes, obesity, hypertension and dyslipidaemia. Although these correlations were not examined in the present study, in our sample the average weight of patients was $86.35 \mathrm{~kg}$, the most significant co-morbidity factors being hyperlipidaemia and hypertension, while the mean duration of diabetes was approximately 10 years. Therefore, these factors are also likely to contribute to the low quality of life of diabetic patients in the present study.

Similar to our results, Zioga et al. [13] also found low life expectancy for diabetic patients in Greece, and the SF-36 scale was used in this study to assess the quality of life of diabetic patients. Our findings are also consistent with many previous studies conducted on diabetic patients in other countries, which indicate that this population, in general, is likely to report lower quality of life-related to physical and mental health compared to the general population [18]-[21] also reported lower quality of life for elderly diabetic patients, as found in the present study, as the mean age of the sample was approximately 65 years. The duration of diabetes, the complexity of treatment, the development of complications, and the challenges to diabetes selfcare often contribute significantly to lower quality of life in these patients.

Concerning the relationship between adherence to antidepressant treatment and quality of life, the results of the present study showed that among all 
dimensions of antidepressant compliance, only personal behavioural attitudes and care attainment were measured. Care of lower limbs were positively correlated with quality of life-related to physical health, while no other correlations between other dimensions of adherence to antidiabetic treatment and quality of life were found that physical and/or mental health [13] also found a correlation between quality of life and compliance in foot care, but also found statistically significant correlations between all dimensions of diabetes treatment compliance (diet, exercise, and blood sugar measurement). blood, self-care recommendations) and all dimensions of quality of life, as assessed by the SF36 scale, but were not identified in the present study due to possible differences in the samples between the two studies. Similarly, previous research performed worldwide has shown that compliance with antidiabetic treatment is associated with increased levels of quality of life [22], [23]. However, the present study did not find any such associations. It is possible that other factors may contribute to the quality of life of sample diabetes patients, such as complications of diabetes, type of medication, or duration of diabetes, but these associations were not examined in the present study. Overall the results of the study show that patients with diabetes showed low levels of compliance with diet and high levels of compliance with lower extremity care and adherence to medication. Patients with type 2 diabetes experienced moderate to the low quality of life-related to physical and mental health. Positive correlations were found only between personal attitudes regarding lower extremity care and compliance with lower extremity care with the quality of life-related to physical health.

These results are important and have implications for the promotion of the health of diabetic patients. Physicians need to make comprehensive recommendations to diabetes patients and their families about diabetes self-care and antidiabetic treatment, as well as training in diabetes management. This could lead to improvements in the quality of life of diabetic patients. This study focused on patients in a public hospital and the mean age of the sample was 65 years, which limits the scalability of the findings to the diabetic patient population in Greece. Future research is needed to confirm its findings, as well as to study other factors that are likely to contribute to the low quality of life of diabetic patients in Greece. Thus, the necessity of a National study in Greece is profound.

\section{References:}

[1] WHO, "Definition and Diagnosis of Diabetes Mellitus and Intermediate Hyperglycemia: report of a WHO/IDF consultation," 2006.

[2] G. E. Umpierrez, "Diabetic Ketoacidosis," in The Diabetes Textbook, Cham: Springer International Publishing, 2019, pp. 619-627.

[3] P. Choudhary, F. Campbell, N. Joule, and P. Kar, "A Type 1 di abetes technology pathway: consensus statement for the use of technology in Type $1 \mathrm{~d}$ iabetes," Diabet. Med., vol. 36, no. 5, pp. 531-538, May 2019.

[4] G. Intas et al., "Development and validation of a d iabetes self-care activities questionnaire," Journal of Diabetes Nursing, vol. 16, no. 3. pp. 100-110, 2012.

[5] F. Rafii, N. S. Fatemi, E. Danielson, C. M. Johansson, and M. Modanloo, "Compliance to treatment in patients with chronic illness: A concept exploration.," Iran. J. Nurs. Midwifery Res., vol. 19, no. 2, pp. 159-67, Mar. 2014.

[6] W. Polonsky, R. H.-P. preference and adherence, and unde fined 2016, " Poor medication adherence in type 2 diabetes: recognizing the scope of the problem and its key contributors," ncbi.nlm.nih.gov.

[7] B. M. Y. E. Cheung, K. L. Ong, S. S. Cherny, P.-C. Sham, A. W. K. Tso, and K. S. L. Lam, "Diabetes Prevalence and Therapeutic Target Achievement in the United States, 1999 to 2006," J. Med., vol. 122, pp. 443-453, 2009.

[8] M. DiBonaventura, N. Wintfeld, and J. Huang, "The association between nonadherence and glycated hemoglobin among type 2 di abetes patients using basal insulin analogs," Patient Prefer. Adherence, vol. 8, pp. 873-882, 2014.

[9] D. Giugliano, M. I. Maiorino, G. Bellastella, and K. Esposito, "Glycemic control in type 2 diabetes: from medication nonadherence to residual vascular risk," Endocrine, vol. 61, no. 1, pp. 23-27, Jul. 2018.

[10] F. Saleh, S. J. Mumu, F. Ara, M. A. Hafez, and L. Ali, "Non-adherence to self-care practices \& medication and health related quality of life among patients with type 2 diabetes: A cross-sectional study," BMC Public Health, vol. 14, no. 1, May 2014.

[11] M. Butt, A. M. Ali, and M. M. Bakry, "Health-related quality of life in poorly controlled type 2 diabetes patientsassociation of patients' characteristics with EQ-5D domains, mean EQ-5D scores, and 
visaul analog scale score," Asian J. Pharm. Clin. Res., vol. 11, no. 1, pp. $93-98$, Jan. 2018.

[12] I. Avramopoulos, "Glycaemic control, treatment satisfaction and quality of life in type $2 \mathrm{~d}$ iabetes patients in Greece: The PANORAMA study Greek results," World J. Diabetes, vol. 6, no. 1, p. 208, 2015.

[13] E. Zioga et al., "Adherence and Quality of Life in Patients With Type II Diabetes Mellitus in Northern Greece," Mater. Socio Medica, vol. 28, no. 4, p. 258, 2016.

[14] Y. V. Martínez, C. A. Prado-Aguilar, R. A. Rascón-Pacheco, and J. J. Valdivia-Martínez, "Quality of life associated with treatment adherence in patients with type 2 diabetes: A cross-sectional study," BMC Health Serv. Res., vol. 8, 2008.

[15] T. A. Saritchai Predawan, Korrakot Sinprajakpol, "Rule Discovery for Diabetes Mellitus Diagnosis using Ant-Miner Algorithm," WSEAS Trans. Biol. Biomed., vol. 16, pp. 61-68, 2019.

[16] G. Makanda, "A Mathematical Model on the Effect of Non-Adherence to Drugs on Diabetes Control," Int. J. Biol. Biomed. Eng., vol. 13, pp. 70-77, 2019.

[17] A. A. Papadopoulos, N. Kontodimopoulos, A. Frydas, E. Ikonomakis, and D. Niakas, "Predictors of health-related quality of life in type II diabetic patients in Greece," BMC Public Health, vol. 7, no. 1, p. 18 6, Dec. 2007.

[18] S. K. Verma et al., "Impact of depression on health related quality of life in patients with diabetes," Ann. Acad. Med. Singapore, vol. 39, no. 12, pp. 913-919, Dec. 2010.

[19] O. Solli, K. Stavem, and I. S. Kristiansen, "Health-related quality of life in diabetes: The associations of complications with EQ5D scores," Health Qual. Life Outcomes, vol. 8, no. 1, p. 18, 2010.

[20] A. J. Green, K. M. Fox, and S. Grandy, "Self-reported hypoglycemia and impact on quality of life and depression among adults with type 2 diabetes mellitus," Diabetes Res. Clin. Pract., vol. 96, no. 3, pp. 313-318, Jun. 2012.

[21] A. A. Kiadaliri, B. Najafi, and M. MirmalekSani, "Quality of life in people with diabetes: A systematic review of studies in Iran," Journal of Diabetes and Metabolic Disorders, vol. 12, no. 1. BioMed Central Ltd., 19-Dec-2013.

[22] S. D. Alfian, H. Sukandar, K. Lestari, and R.
Abdulah, "Medication Adherence Contributes to an Improved Quality of Life in Type 2 Diabetes Mellitus Patients: A Cross-Sectional Study," Diabetes Ther., vol. 7, no. 4, pp. 755-764, Dec. 2016.

[23] F. S. Marinho, C. B. M. Moram, P. C. Rodrigues, N. C. Leite, G. F. Salles, and C. R. L. Cardoso, "Treatment adherence and its associated factors in patients with type 2 diabetes: Results from the Rio de Janeiro type $2 \mathrm{~d}$ iabetes cohort study," J. Diabetes Res., vol. 2018, p. 8, 2018. 